\title{
"Who is on my side?" When the investigator feels that the IACUC is working against them
}

\author{
ecause Joseph Kleiner is employed \\ by the university, he is responsible \\ for making sure that all the rules \\ and regulations are followed. While it is \\ understandable that Dr. Macdonald might \\ feel like the university is trying to intimidate \\ him by surprising him with a lawyer present \\ at the meeting, Kleiner should be able to \\ adequately represent both the school's and \\ Dr. Macdonald's best interests. Kleiner is \\ involved in the meeting to be an advocate \\ for Dr. Macdonald and will likely argue \\ for him because he is employed by the \\ university and any disciplinary actions \\ taken against Dr. Macdonald could reflect \\ poorly on the school. The chairman of \\ the committee should have prepared \\ Dr. Macdonald for this meeting by informing \\ him who would be present as well as
}

clarifying Kleiner's role as a non-voting observer within the committee, and what that means (i.e. legal representation); moreover, he is not there to intimidate him. Dr. Macdonald may also be concerned about confidential material leaving the room since Kleiner is not an actual member of the IACUC. The committee should assure Dr. Macdonald that nothing that is discussed in the meeting will be shared with anyone outside of the IACUC.

Since this is a non-compliance issue that needs to be resolved in a timely manner, the IACUC can give Dr. Macdonald the option to procure his own lawyer, if he so chooses. However, it should be explained to him that this will only delay the entire process because all non-approved animal work will be suspended until the amendment goes through and the non-compliance issue is resolved. If he chooses to obtain his own lawyer, that should be something he is required to pay for since Kleiner, in a way, was the lawyer the university provided for him.

The IACUC and Dr. Macdonald appear to have started off on the wrong foot in this scenario. However, this relationship can be salvaged by transparent communication from here on out.

\section{Jui Tu, Leo Holguin and Yvonne R Cornejo* Center for Comparative Medicine, City of Hope Beckman Research Institute, Duarte, California, USA. \\ *e-mail:yvcornejo@coh.org}

Published online: 24 July 2018

https://doi.org/10.1038/s41684-018-0110-z

\section{IACUC is in the wrong}

T每 his case is a series of mishandlings on the part of the IACUC, which led to this unpleasant situation.

Beginning with the first mistake, before the meeting, the IACUC chairman should have conducted a more thorough investigation of the allegations against Dr Macdonald. There is a big difference between starting an experiment without approval and adding extra animals to a previously approved protocol. The first scenario is very serious. How could a scientist start a project without any approval? This kind of investigation should include the necessary assistance to understand exactly what happened. In a few words, the IACUC chairman should explore, in depth, the case before inviting the scientist to explain. Of course, in either case there is no excuse for Macdonald. However, what the subcommittee should investigate and clarify is if Macdonald ignored the IACUC intentionally or if he didn't understand committee procedures.

The second mistake was that Macdonald wasn't informed beforehand about the composition of the subcommittee. Macdonald's reaction to the presence of attorney Kleiner was reasonable. He saw a lawyer as a member of the subcommittee; it doesn't matter if he is a voting or non-voting member. So, it is normal for Macdonald to consider himself an "accused person". The IACUC sent the wrong message. In addition, the explanations provided from the IACUC chairman are far from reality. Given that Kleiner is the school's attorney and Macdonald has to explain his actions, which violated school policy, it is clear there is a serious conflict and that Kleiner can't represent both parties. Under these circumstances, it is logical for Macdonald to ask for his own lawyer and he has the right to do so. This complicated situation could have been avoided if Kleiner had a permanent position as a layperson on the IACUC and Macdonald been informed.

Now, the Chairman of the IACUC has two choices: either to allow Macdonald to have his own legal representative or to proceed without the participation of attorney Kleiner. The first option gives the impression that the IACUC is a court. This is not desirable for the role as well as the reputation of the IACUC. The second choice seems preferable; however, there is a serious risk of devaluing the role of Kleiner within the subcommittee and of course the committee itself. Thus, despite the potential negative effects, the first option looks more appropriate. Macdonald should be allowed representation by his own lawyer.

The important issues/messages, which are raised from this case, are related to the IACUC's role and policies and how these are communicated to the scientific community. Is the IACUC a court for those breaking the law? Is the IACUC a regulatory, surveillance, and advisory body aiming to guarantee the proper care and use of animals as well as execution of good science? Is the composition of the IACUC known to the scientific community? These are some of the questions, which must be answered if we want to guarantee the reliability and the transparency of the IACUC.

Nikolaos Kostomitsopoulos

Biomedical Research Foundation of the Academy of

Athens (BRFAA), Athens, Greece.

e-mail:nkostom@bioacademy.gr

Published online: 24 July 2018

https://doi.org/10.1038/s41684-018-0111-y 